\title{
Educating junior doctors on DNACPR and ceiling of treatment policy improves compliance with form completion and documentation of resuscitation decisions
}

\author{
Authors: Liga Ermane ${ }^{\mathrm{A}}$ and Harith Altemimi ${ }^{\mathrm{A}}$
}

\section{Introduction}

Do not attempt cardiopulmonary resuscitation (DNACPR) orders are important in avoiding inappropriate CPR attempts. Clear and full documentation of decisions about CPR, the reasons for them, and the discussions with those involved in the decisions, is an essential part of high-quality care. ${ }^{1}$ A new combined Queen Elizabeth Hospital King's Lynn DNACPR and Ceiling of Treatment form was implemented in July 2018. A new audit tool was developed by the resuscitation department to ensure accurate completion of DNACPR forms was captured. Subsequent re-audits did not show any significant improvement in compliance with set standards and in some cases showed deterioration. Therefore, in January 2019 teaching sessions on the importance of DNACPR and Ceiling of Treatment documentation were introduced.

\section{Materials and methods}

Teaching sessions were delivered to junior doctors in small and large group settings between 10 January 2019 and 10 June 2019. Each session was around 10-15 minutes long. Awareness of the audit tool used in monthly audits was raised during the teaching sessions. DNACPR and Ceiling of Treatment orders and mental capacity assessment forms were explored, and accurate documentation was discussed. Prospective evaluation of teaching session effectiveness was done by reviewing monthly re-audit results on DNACPR and Ceiling of Treatment documentation performed by the Resuscitation department.

\section{Results and discussion}

In total 101 (76\%) junior doctors from a wide range of specialties were educated on DNACPR documentation. There has been a significant improvement in overall compliance with DNACPR and Ceiling of Treatment order documentation, rising from $84 \%$ in January to $90 \%$ in June (Fig 1). There was a marked improvement in decision making and documentation of Ceiling of Treatment increasing from $73 \%$ in January to $86 \%$ in June (Fig 2). Improvements were also noticed in other standards, including documented decisions and discussions in forms and medical notes,

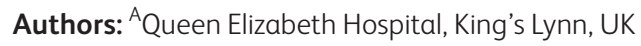

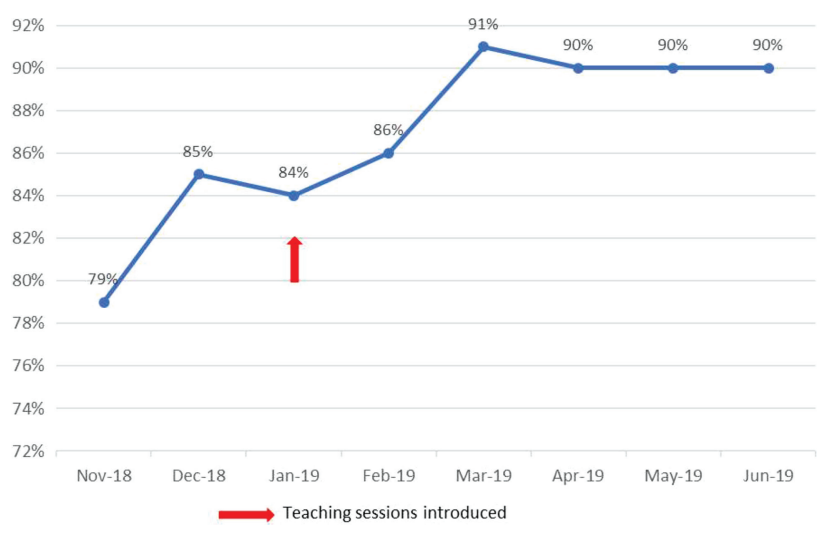

Fig 1. Overall compliance with do not attempt cardiopulmonary resuscitation documentation.

forms being signed by appropriate team members, and clearly recorded indefinite decisions. Compliance with mental capacity assessment has been poor most of the time and was worse in June (39\%) than in January (65\%); however, May results showed overall compliance in this standard of $87 \%$. We expect that these metrics can be improved even further with continued training and education of the doctors at all levels.

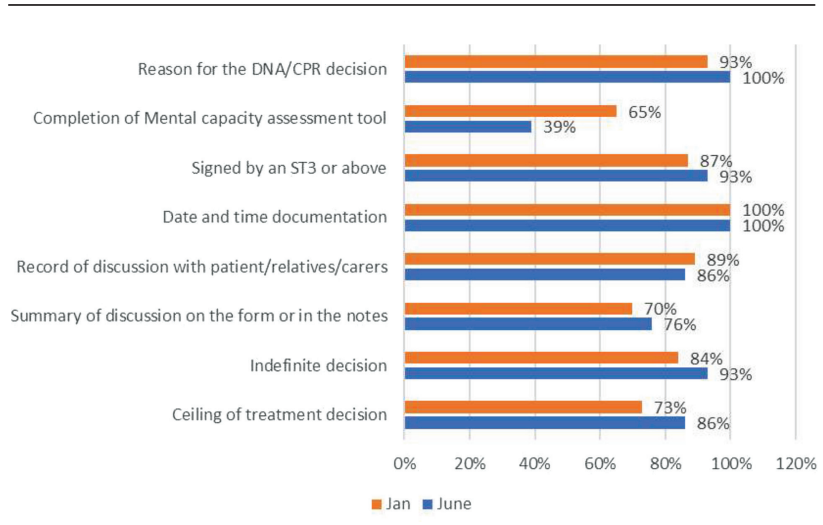

Fig 2. Compliance with standards. DNA/CPR = do not attempt cardiopulmonary resuscitation. 


\section{Conclusion}

Accurate documentation of DNACPR and Ceiling of Treatment forms is crucial for high-quality care. Correct completion of these orders can be improved by effective teaching sessions. There is room for improvement in all standards especially in mental capacity assessments.

\section{Conflicts of interest}

None declared.

\section{Reference}

1 British Medical Association, Resuscitation Council (UK), Royal College of Nursing. Decisions relating to cardiopulmonary resuscitation (3rd edition - 1st revision). RC(UK), 2016. www.resus. org.uk/dnacpr/decisions-relating-to-cpr [Accessed 27 October 2019]. 